\title{
Les limites du récit
}

\author{
Jérôme Porée \\ Université de Rennes I
}

\section{Résumé:}

Les notions de "temps raconté" et d"identité narrative" sont devenues, en moins de trois décennies, des lieux communs non seulement pour le philosophe mais encore pour le psychologue et l'éthicien. On devrait s'en réjouir si elles n'alimentaient souvent, aujourd'hui, ce qu'il faut bien appeler un nouveau dogmatisme. Or si Paul Ricœur a bien affirmé, de plusieurs façons, les ressources du récit, il a été aussi le premier à reconnaître ses limites - des limites dont on peut se demander si elles ne sont pas, plus généralement, celles de l'herméneutique elle-même.

Mots-clés: Récit, Temps, Souffrance, Psychanalyse, Phénoménologie

\begin{abstract}
:
The notions of "narrated time" and "narrative identity" have become, in less than three decades, commonplaces, not only for philosophers, but also for psychologists and ethicists. This would be welcomed, if only it were not used nowadays in what must be called a new dogmatic way. Now, Paul Ricœur indeed asserted, in various ways, the wealth of the notion of narrative; but he also readily acknowleged its limits aren't these limits those of hermeneutics itself?
\end{abstract}

Key-words: Narrative, Time, Suffering, Psychoanalysis, Phenomenology

Études Ricœuriennes / Ricœur Studies, Vol 4, No 2 (2013), pp.38-49

ISSN 2155-1162 (online) DOI 10.5195/errs.2013.178

http://ricoeur.pitt.edu

\section{(c)) EY-NC-ND}

This work is licensed under a Creative Commons Attribution-Noncommercial-No Derivative Works 3.0 United States License.

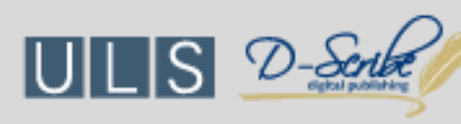

This journal is published by the University Library System of the University of Pittsburgh as part of its D-Scribe Digital Publishing Program, and is cosponsored by the University of Pittsburgh Press. 


\title{
Les limites du récit
}

\author{
Jérôme Porée \\ Université de Rennes I
}

Paul Ricœur est regardé à bon droit comme un penseur du récit. Les notions de "temps raconté" et d'identité narrative" sont devenues, en moins de trois décennies, des lieux communs non seulement pour le philosophe mais encore pour le psychologue et l'éthicien. On devrait s'en réjouir si ces notions n'étaient souvent détournées de leur sens et associées à ce qui apparaît aujourd'hui comme une étonnante - et problématique - inflation du récit.

Nous avons en vue non, ce disant, la mode américaine du "storytelling," qui met la narration au service de stratégies commerciales qui n'en retiennent que la puissance d'illusion, ${ }^{1}$ mais des usages du récit qui ne peuvent pas être suspectés d'une telle tromperie et qui sont précisément ceux auxquels se réfèrent ceux que nous venons de nommer. "Racontez et vous serez sauvés!," semblent-ils dire de concert. Ils seront bientôt rejoints par le médecin si, d'aventure, l'Evidence Based Medicine laisse la place à la Narrative Based Medicine et si l'art de raconter devient une branche de l'art de soigner - comme il l'est déjà pour certains professeurs de la faculté de médecine de Columbia qui ont récemment promu la narratologie matière d'enseignement fondamentale, à côté de la physiologie et de l'anatomie. Or Ricœur a bien affirmé, de plusieurs façons, les ressources du récit, mais il a été aussi le premier à reconnaître ses limites.

Ces limites sont de deux sortes. Vers le haut, elles reconduisent à d'autres modalités du langage et notamment à l'argumentation, avec laquelle la narration est appelée à nouer, en philosophie comme en politique, une relation circulaire. Il n'est qu'à évoquer ici le rôle conciliateur joué par Ricœur, dès les années soixante-dix, dans le débat entre l'herméneutique et la critique des idéologies; ce débat préfigurait celui qu'il eut vingt ans plus tard avec Jean-Marc Ferry, adepte, comme Habermas, d'une philosophie "reconstructive," ${ }^{2}$ c'est-à-dire d'une philosophie qui articule plusieurs niveaux de discours - la narration appelant l'interprétation, qui tire elle-même ses raisons de l'argumentation, avec laquelle seulement elle peut honorer sa prétention à la vérité. Non que Ricœur hiérarchise de la même façon ces niveaux de discours; il concède seulement que la narration - dont il envisage à ce moment le rôle dans la formation des traditions - a besoin de la critique. ${ }^{3}$ Mais cette concession est décisive. Elle montre que le récit ne suffit pas au récit. Qu'il s'agisse de la possibilité du discours vrai ou de l'émancipation des individus et des groupes sociaux, l'art de raconter ne remplace pas la discussion argumentée.

Vers le bas, les limites du récit sont celles de son rôle dans la constitution du temps et de l'identité personnelle. Nous pourrions nous contenter ici de citer Ricœur au moment où il introduisait pour la première fois cette notion: "l'identité narrative n'épuise pas [...] l'ipséité du sujet." ${ }^{4}$ Cette proposition, si on la prend au sérieux, oblige à joindre à l'identité narrative les "composantes non narratives" de notre personne. ${ }^{5}$ Cette obligation est renforcée par le fait que la narration est une catégorie de l'action, avec laquelle elle a une parenté que vérifient également l'histoire et le mythe. ${ }^{6}$ Car l'homme est un être "agissant et souffrant." Non que la souffrance ellemême ne "demande récit"; mais, à la différence de la faute, qui trouve dans l'aveu une expression à sa mesure, elle reste proprement inénarrable. Elle mobilise donc des ressources qui viennent de plus loin que tout récit. Quelles ressources? Cette question est l'enjeu véritable du dialogue de 
Ricœur avec la psychanalyse, où pourtant il trouve le premier modèle de l'identité narrative. Elle est aussi l'occasion de se rappeler que la phénoménologie n'est pas seulement, pour lui, une étape sur le chemin de l'herméneutique, mais son "indépassable présupposition." Des deux sortes de limites du récit que nous venons de distinguer, nous considérerons surtout les secondes.

Il nous faudra faire d'abord, pour cela, de brefs rappels concernant les notions conjointes de "temps raconté" et d"'identité narrative" (1). Nous dirons ensuite pourquoi ces notions, pour Ricœur lui-même, restent problématiques - pourquoi donc il ne faut pas en faire un usage dogmatique (2). Enfin nous développerons les questions ébauchées à l'instant à propos de la souffrance et nous nous demanderons si les limites du récit ne sont pas, plus généralement, celles de l'herméneutique elle-même (3)

\section{Temps raconté, identité narrative}

On connaît la thèse défendue par Thierry Hentsch dans un ouvrage au titre suggestif ${ }^{8}$ : nous racontons pour ne pas mourir - enfin, pour ne pas mourir tout à fait... Comment ne pas songer alors à l'antique représentation du temps comme un dieu qui dévore ses propres enfants? Le récit est peut-être le meilleur moyen qu'ont trouvé ces derniers pour échapper à sa voracité. Il faut cependant, cela admis, distinguer entre plusieurs modes du vivre, comme entre plusieurs modalités du raconter. Le livre de Hentsch est consacré aux grands récits qui irriguent en profondeur nos traditions et nos croyances. Transmis de génération en génération, ces grands récits surmontent la discontinuité du temps biologique et nouent le fil d'un temps que l'on peut appeler, par contraste, le temps humain. On pourrait se demander, certes, si ce fil n'est pas, aujourd'hui, irrémédiablement brisé et si notre expérience la plus commune n'est pas plutôt celle d'un temps discontinu. Mais il n'y a pas seulement les grands récits: il y a encore la narration quotidienne de nos plus humbles expériences. Entre ces deux limites prennent place mille et une manières de raconter. Deux lignes de partage, parmi d'autres, peuvent être tracées dans le vaste champ ainsi ouvert. La première passe précisément entre la narration spontanée de l'expérience quotidienne et le récit organisé selon des règles qui permettent d'en parler comme d'un art ${ }^{9}$; la seconde oppose le récit historique et le récit de fiction. Ces lignes ne sont pas infranchissables. Il existe, entre les différentes modalités du raconter, des liens nombreux et complexes. ${ }^{10}$ Nous n'examinerons pas tous ces liens - nous contentant d'indiquer comment le récit, dans ses diverses modalités, permet à l'homme d'assumer sa condition temporelle, et nous fondant pour cela sur les livres jumeaux que sont Temps et récit et Soi-même comme un autre, dont nous ne dirons cependant rien de plus que ce qui est utile à notre propos.

La tonalité existentielle de la réflexion est justifiée, s'agissant de Ricœur, par le fait que le temps n'est pas rencontré d'abord comme un problème d'ordre théorique, mais comme une question qui inquiète en chaque homme son "désir d'être." Non que cette question ne puisse être élaborée en problème: une telle problématisation est l'œuvre propre du discours philosophique; elle explique la place que trouvent, dans Temps et récit, les théories de saint Augustin, de Kant, de Husserl ou de Heidegger... Mais ces théories trouvent vite leur limite - et que dire de leur intérêt pour la vie? Le plan du troisième tome de l'ouvrage est, à cet égard, parfaitement clair: à "l'aporétique de la temporalité," telle que l'élabore le discours philosophique, répond une "poétique du récit" dans laquelle ce mot: "poétique," désigne un ensemble de ressources propres à soutenir notre désir d'être ou ce qui apparaîtra plus proprement alors - la nuance est importante - comme notre "effort pour exister." 
La thèse générale de Ricœur est qu'il y a une "connexion significative" entre la fonction narrative et l'expérience humaine du temps. C'est qu'aucun récit ne se réduit au simple constat qu'une chose arrive, puis une autre, puis encore une autre... A l'intérieur de cette multiplicité, il introduit une unité que l'on peut comprendre, à la suite d'Aristote, comme l'unité d'une intrigue. Dans sa Poétique, en effet, Aristote s'était penché sur le pouvoir singulier de la tragédie, entendue comme une espèce de poésie narrative. Il s'était demandé comment la haine, la vengeance, la souffrance et la guerre pouvaient recevoir dans la narration qu'en fait le poète une valeur opposée à celle que nous leur attribuons dans la vie réelle. La difficulté était d'autant plus grande qu'il avait commencé par définir la tragédie comme une représentation des actions humaines. Comment, en effet, pourrait-il y avoir davantage, dans la représentation de la réalité, que dans la réalité qu'elle représente? Mais on suppose alors, entre l'une et l'autre, un rapport de copie à modèle. Or ce n'est pas le sens qu'Aristote donne au mot mimèsis, que nous traduisons par représentation. La mimèsis tragique n'est pas une simple duplication de la réalité. Elle opère une mise à distance qui permet à celle-ci d'apparaître dans une lumière neuve. Cette mise à distance est l'œuvre du muthos, c'est-à-dire de l'histoire ou, justement, de l'intrigue inventée par le poète. Le muthos est la condition de la mimèsis. Grâce à lui, les actions humaines peuvent apparaître dans la tragédie autrement qu'elles n'apparaissent dans la vie réelle. D'un côté, certes, ce sont les mêmes et le spectateur peut s'en émouvoir; mais, d'un autre côté, elles sont différentes et il peut les contempler sans répulsion. Lecteur d'Aristote, Ricœur lui emprunte cette notion d'intrigue. Il l'étend cependant à toutes sortes de récits; et il met l'accent surtout sur son pouvoir d'agencement ou de réagencement de la réalité. C'est qu'il s'agit toujours, en racontant, de "riposter" au désordre du monde ou de nos vies. L'intrigue est l'âme du récit. Elle met en relation les différents événements qui le composent. Au cours épisodique de leur succession, elle superpose l'ordre logique d'une "configuration." Le récit opère ainsi une synthèse du temps. D'une suite de moments quelconques, il fait une histoire sensée.

Ces propositions ont pour contrepoint la conception augustinienne du temps comme "distension de l'âme." Car dans cette conception, remarque Ricœur, "la discordance ne cesse de démentir le vœu de concordance constitutif de l'animus." ${ }^{11}$ Aussi ce vœu ne peut-il être réalisé qu'au-delà du temps. L'analyse aristotélicienne de la mimèsis tragique va dans l'autre sens: elle établit "la primauté de la concordance sur la discordance dans la configuration de l'intrigue." Le temps peut être défini ainsi comme une "concordance discordante." ${ }^{\prime 12}$ Cette définition est vérifiée autant par le récit historique que par le récit de fiction. Elle l'est aussi, dans une certaine mesure, par la narration spontanée de la vie quotidienne.

C'est le point cependant où la problématique du temps raconté rejoint celle de l'identité personnelle. Car l'identité des personnes n'est pas, comme celle des choses, une identité substantielle: c'est une identité temporelle. Aussi est-elle constamment menacée de se perdre dans la succession d'états momentanés et sans liens entre eux. On peut se demander alors comment elle peut être assurée de rester la même. La question est compliquée par le fait qu'elle conjugue deux traits: la "mêmeté" et la "mienneté," la permanence et l'ipséité. Car il faut comprendre comment ces traits peuvent être articulés entre eux. La réponse tient précisément dans la notion d'identité narrative. Cette notion lie, en effet, notre capacité d'être nous-mêmes et celle de raconter une histoire dans laquelle nous puissions nous reconnaitre. ${ }^{13}$ Ce qui est en jeu cependant dans la constitution de l'identité personnelle est moins la "configuration" que la "refiguration" du temps par le récit. Cette refiguration fait de notre vie la résultante de toutes les histoires véridiques ou fictives que nous racontons à son propos. Appelée par les changements liés à notre situation, elle a le sens d'une reprise de soi par soi. 
Configuration, refiguration... - il est nécessaire cependant de compliquer encore le modèle tiré de la conception aristotélicienne de la mimèsis, que Ricœur comprend, on le sait, comme une opération triple. Car les histoires que nous racontons ne naissent pas de rien: elles émergent sur un fond d'histoires déjà racontées. Il faut bien d'ailleurs que nos vies, non seulement demandent, mais encore se prêtent à être racontées. On peut parler en ce sens de la "structure pré-narrative" de l'expérience humaine en général. Ce que l'intrigue configure selon les règles propres de l'art de raconter est "préfiguré" dans une sorte de narrativité inchoative. Si l'on songe à l'expérience pratique, qui est l'objet privilégié de la Poétique d'Aristote, cela revient à dire que l'action est une narration qui s'ignore encore.

Ajoutons seulement, à ces brèves remarques, que l'identité narrative n'est pas celle d'un soi isolé. Car le récit, d'une part, compose la permanence et le changement, mais il est toujours, d'autre part, un récit à plusieurs voix. A la dialectique de l'ipséité et de la mêmeté, se superpose donc la dialectique de l'ipséité et de l'altérité. C'est le sens le plus général de ce titre: Soi-même comme un autre.

\section{Quatre limites du récit}

Sans épiloguer sur cette double dialectique, nous relèverons à présent quatre limites $\mathrm{du}$ récit. Nous déplacerons progressivement l'accent, pour ce faire, de l'agir au souffrir. C'est sur ce dernier plan, en effet, que ces limites sont les plus criantes.

a) On peut parler, pour la première, d'une limite interne. C'est une remarque souvent formulée par Ricœur: il n'y a pas de récit total, d' "intrigue de toutes les intrigues." L'unité introduite par le récit dans la multiplicité de l'expérience temporelle est une unité "partielle" et "plurielle." ${ }^{14}$ Cette remarque est dirigée d'abord contre la prétention de la philosophie hégélienne de l'histoire: celle d'englober dans un même savoir, ou de compter comme autant d'étapes d'un même progrès, les figures diverses de notre humanité. Un récit total impliquerait une médiation totale. Or la médiation opérée par le récit est toujours imparfaite et inachevée. ${ }^{15}$ On pourrait se demander cependant si le récit, au nom duquel est dénoncée cette illusion totalisante, n'entretient pas lui-même cette illusion. C'est le cas certainement du mythe, tant qu'il ne reçoit pas l'éclairage de la critique. Ricœur en fait la remarque à propos des mythes de salut ${ }^{16}$, qu'il tient pour les répondants des mythes d'origine dont il dresse la typologie dans La symbolique du mal. Il ne manque pas d'ailleurs de les mettre en relation avec la conception moderne du progrès - un progrès tenu alors pour inéluctable. Comment faire pour que l'ordre du récit ne joue pas dans le mythe un rôle analogue à celui de la raison dans les grands systèmes spéculatifs? Telle est, à ce moment, la question posée. C'est la tâche même, répond Ricœur, d'une herméneutique critique. Mais la critique alors n'est pas l'œuvre du récit. Elle ressortit à d'autres modalités du langage et de la communication.

La même remarque peut être appliquée à la compréhension narrative de soi. L'idée que l'unité du récit est une unité partielle et plurielle n'est pas dirigée seulement, en effet, contre la philosophie spéculative. Elle doit être opposée encore à la tendance qu'a chaque personne, comme chaque communauté, à se fermer sur elle-même. Cette tendance la conduit à ignorer ses multiples héritages. Elle lui masque le fait qu'elle est elle-même le produit non d'une mais de plusieurs histoires. C'est parce qu'il n'y a pas de récit total que nous sommes, comme l'écrit Ricœur, en conflit et en négociation avec nous-mêmes. ${ }^{17}$ Mais ces conflits nés du récit, encore une fois, il n'appartient pas au récit de les résoudre. Encore moins sait-il différencier, parmi nos multiples héritages, ceux qui méritent et ceux qui ne méritent pas d'être transmis. On pourrait en 
effet opposer, en simplifiant, deux espèces de récits ${ }^{18}$ : les uns attachent l'individu à son groupe, à sa caste, à son clan; les autres mettent l'homme en relation avec l'homme; les uns narrent le crime et l'expiation, ils nomment l'ennemi héréditaire, ils désignent les victimes sacrifiées à l'utilité commune; les autres invitent au don, ils enseignent l'hospitalité, ils commandent l'accueil du visage étranger. Mais comment distinguer les uns des autres? La compréhension narrative de soi manque, par elle-même, du critère qui lui permettrait d'opérer cette distinction. C'est en ce sens que nous disions en commençant, à propos du débat de Ricœur avec Habermas et Ferry: le récit ne suffit pas au récit.

Il est cependant d'autres limites du récit. Si la première est une limite interne, on peut parler, pour ces dernières, de limites externes. Nous en considérerons trois.

b) La première est liée à notre modernité. L'âge de la science et de la technique est aussi, en effet, celui de la crise du récit, qui n'est elle-même que l'aspect le plus apparent d'une crise générale du langage et de la culture. En 1938, dans un article prophétique ${ }^{19}$, Walter Benjamin écrivait à ce propos: "l'art de raconter est en train de se perdre"; et il déplorait que se perdît ainsi notre faculté d'"échanger des expériences." La narration, montrait-il, inscrit cet échange dans la durée. Or elle est éclipsée désormais par l'information, qui n'a de valeur qu'"au temps de sa nouveauté." Un nouvel homme voit ainsi le jour, que l'on peut appeler l'homme de l'instant. Le roman, objectera-t-on, se porte bien. Mais il est pour Benjamin le symptôme de la crise de la narration dans ses formes traditionnelles. C'est l'œuvre d'un individu solitaire qui s'adresse à d'autres individus solitaires. Cette narration, justement, ne fait plus tradition. Qu'eût-il dit alors de la vogue actuelle de la biographie et du récit de soi? L'enjeu de la crise de la narration, dans tous les cas, n'est pas seulement esthétique; il est encore - et d'abord - anthropologique. C'est bien ainsi que l'entend Benjamin, qui ne sépare jamais la capacité personnelle de raconter, des formes narratives instituées dans une culture donnée. Cette capacité fait fond, on l'a dit, sur des histoires déjà racontées, qui opèrent au niveau de la préfiguration du temps et offrent à chacun des ressources de symbolisation qui l'aident à configurer et à refigurer sa propre vie. Or on peut craindre que ces ressources, aujourd'hui, soient épuisées. Le récit de soi, dès lors, tourne à vide: il n'a plus le pouvoir d'unifier le divers de l'expérience. Cette crainte, il est vrai, n'a jamais profondément troublé Ricœur. Il se méfiait, d'une manière générale, des prophéties autoréalisatrices. Aussi ne trouve-t-on chez lui rien d'équivalent à la critique heideggérienne de la technique. Il ne croyait pas que celle-ci pût menacer radicalement l'institution du signe et - moins encore - l'essence parlante de l'homme. La crise de la narration, telle qu'il l'entend, ne signifie donc pas sa fin; elle n'empêche pas l'homme de rester, fondamentalement, un animal symbolique. Nous n'avons, d'ailleurs, "aucune idée de ce que serait une culture où l'on ne saurait plus ce que signifie raconter." ${ }^{20}$ Nous n'avons aucune idée non plus de ce que serait une culture où l'identité des personnes ne serait pas une identité narrative.

c) Mais ici s'impose une autre limite. Admettons, par hypothèse, que l'identité personnelle soit fondamentament une identité narrative. Cela n'empêche pas de se demander si certains événements ne restent pas réfractaires à la synthèse opérée par le récit. Dans son livre, Paroles suffoquées, Sarah Kofman dit comment certains rescapés des camps des camps de la mort n'avaient de cesse de raconter, raconter sans fin, "comme si seul un [récit] infini était à la mesure du dénuement infini." ${ }^{21}$ Primo Lévi l'affirme à son tour: "le besoin de raconter [...] avait acquis chez nous, avant comme après notre libération, la force d'une impulsion immédiate, aussi impérieuse que les autres besoins élémentaires." Mais au besoin de raconter s'opposait, chez d'autres rescapés, la certitude de n'être pas crus; c'est ce qui a conduit le plus grand nombre au silence. Lévi parle à ce propos de "rêve de récit." Ce rêve de récit ne résistait pas à l'afflux 
d'"images plus indistinctes" où entraient pêle-mêle la faim, les coups, les cris, le froid, la saleté, la nausée, la fatigue, la peur, l'horreur, l'insomnie et les nuits de fièvre. Comment, en effet, mettre de l'ordre dans ce désordre, sans méconnaître au même moment la réalité qu'il révèle? Et comment surtout faire sienne une telle réalité? Il faudrait, encore une fois, un "récit infini." Mais un tel récit justement n'existe pas; et c'est ce que signifie ce titre: "paroles suffoquées." Ricœur aurait pu l'adopter lorsqu'il revient, dans la dernière étude de Soi-même comme un autre, sur l'identité narrative et remarque, à propos de la thèse élaborée dans les cinquième et sixième études, qu'"il faudrait aller plus loin et prendre en compte des formes plus dissimulées du souffrir: l'incapacité de raconter, le refus de raconter, l'insistance de l'inénarrable, phénomènes qui vont bien au-delà de la péripétie, toujours récupérable au bénéfice du sens par la stratégie de mise en intrigue." ${ }^{\prime 2}$

d) Nous en venons par là à une dernière limite: le débordement du genre narratif par un langage qui fait reculer le point où la méditation du temps s'abîme dans le silence. Il vient en effet un moment où "il n'appartient plus à l'art narratif de déplorer la brièveté de la vie, le conflit de l'amour et de la mort, la vastitude d'un univers qui ignore notre plainte"; non qu'alors, toutes les voix se taisent, mais seule parle encore une voix "qui est aussi un chant" et où l'on peut reconnaître, figure lyrique de la plainte, la "sempiternelle élégie." ${ }^{23}$ Encore vient-il après cela un moment où cette voix aussi se tait. Que reste-t-il alors?

Nous ne répondrons pas directement à cette question. Nous voudrions en effet la situer d'abord en son lieu propre. Or ce lieu n'est autre que la souffrance. Nous n'avons pas sans motif relevé plus haut l'affinité de la narration et de l'action. La mimèsis aristotélicienne déjà est mimèsis praxeos. Il en est de même de la mimèsis telle que la pense Ricœur. Prise au niveau le plus profond, certes, elle l'entraîne à parler de la "structure pré-narrative de l'expérience en général." Mais il n’y a pas une structure pré-narrative de la souffrance comme il y a une structure pré-narrative de l'action. Bien plutôt la souffrance suspend-elle le sens préfiguré par une telle structure. ${ }^{24} \mathrm{C}^{\prime}$ est une fausse symétrie, en ce sens, que celle de l'agir et du souffrir. Non que Ricœur n'affirme leur "corrélation originaire." ${ }^{25}$ Cette corrélation évoque celle du volontaire et de l'involontaire, qui était au centre de la thèse de 1950. Mais, s'il y a une "réciprocité du volontaire et de l'involontaire," il n'y a pas, en revanche, de réciprocité de l'agir et du souffrir. Le souffrir correspond plutôt à ce que Ricœur appelait alors l' "involontaire absolu." L'équivalent de cet involontaire absolu, dans le contexte de son herméneutique du récit, est précisément $1{ }^{1}$ "insistance de l'inénarrable." ${ }^{26}$ Et la question, ici et là, est la même: comment vivre malgré tout? C'est cette question qui explique l'intérêt de Ricœur pour la psychanalyse. Nous dirons en terminant comment il est ramené par ce biais à la phénoménologie et, qui plus est, à une phénoménologie sans herméneutique.

\section{L'épreuve de l'inénarrable}

La psychanalyse accompagne tout le chemin de pensée de Ricœur ${ }^{27}$. On aurait donc tort de borner son intérêt pour celle-ci à l'Essai sur Freud et au problème - intérieur à l'herméneutique - du "conflit des interprétations." On aurait tort aussi de croire que sa relation avec la psychanalyse soit marquée avant tout par l'hostilité ou la polémique. Son appropriation des notions de "travail de mémoire" et de "travail de deuil" en est le meilleur exemple. C'est encore plus vrai si l'on oppose, comme le fait Freud, deuil et mélancolie. Car la mélancolie est ce contre quoi Ricœur n'a cessé de lutter comme personne et comme penseur: elle est la négation de 
$l^{\prime}$ "affirmation originaire," l'inversion de notre "désir d'être," la "culture pure de la pulsion de mort."

La grande découverte de Freud, à ses yeux, et le premier motif de l'inquiétude où elle le jette, est justement celle de la pulsion de mort, ce "principe en nous de toutes les régressions." Si elle n'annule pas l'affirmation de la vie, elle rend cette affirmation même difficile et incertaine. Comment vivre malgré tout? Telle est, encore une fois, la question posée.

Nous n'ignorons pas, certes, les nombreux textes où notre philosophe y répond en mobilisant les ressources du récit: à celui-ci de rendre la vie à la fois "plus supportable" et "plus intelligible." C'est pourquoi la psychanalyse ne fournit pas seulement, au début des années quatre-vingt, le premier modèle de l'identité narrative, mais fait encore elle-même l'objet d'une réévaluation qu'explique précisément le rôle qu'y joue la narrativité ${ }^{28}$ La récente édition, par le Fonds Ricœur, d'"écrits et conférences" autour de la psychanalyse ${ }^{29}$ datés pour la plupart des mêmes années, en témoigne. Dans celui qui va le plus loin dans ce sens, la vie elle-même est définie, en un sens littéral et non métaphorique, comme "un récit en quête de narrateur. ${ }^{\prime 30}$ Le corollaire de cette thèse, $c^{\prime}$ est $q^{\prime}$ ' "une vie n'est qu'un phénomène biologique tant qu'elle n'est pas interprétée" sous le régime de la refiguration narrative. Mais ces déclarations sont amendées par de nombreuses autres.

On peut se rappeler d'abord ce que Ricœur tient, dès l'Essai sur Freud, pour "la question épistémologique de la psychanalyse": celle de la coexistence, dans un même discours, de deux sortes d'énoncés, les uns exprimant des rapports de force justiciables d'une énergétique, les autres articulant des significations justiciables d'une herméneutique. Car un tel discours, certes, est "ambigu" et son épistémologie, une "épistémologie mixte." Mais ce mixte, nous le sommes nous-mêmes. Aussi l'ambiguité affecte-t-elle d'abord dans notre personne le rapport de l'être et du sens, de l'affirmation et de la compréhension, du pouvoir de vivre et du pouvoir de raconter. Il en résulte que si l'homme a vocation au langage, sa vie pourtant n'est pas seulement la vie du langage. Ricœur le rappelle contre les interprétations purement linguistiques de la psychanalyse. ${ }^{31}$ Que reste-t-il de la vie lorsqu' elle n'est plus portée par le langage et en particulier par le récit? Telle est alors la question posée. Si elle justifie la place réservée par Freud à la théorie des pulsions, elle n'explique pas moins les multiples renvois de Ricœur à l' "affirmation originaire." Aussi peut-on penser que c'est le secret d'une telle affirmation que ce dernier cherche à percer dans son dialogue avec la psychanalyse. Objectera-t-on que la pulsion n'existe qu'à travers ses "représentants"? Ricœur lui-même formule cette objection contre le biologisme latent de certains écrits freudiens sur ce thème et il en démarque à l'occasion sa propre conception de l'affirmation originaire. Mais c'est là, justement, l'ambiguïté, c'est là le nœud du problème. D'où la protestation qu'il élève en sens inverse, quelques années plus tard, contre "une conception unilatéralement herméneutique" ${ }^{\prime \prime 2}$ du vivre humain. Il faut l'avouer: le problème épistémologique de la psychanalyse freudienne est aussi le problème anthropologique de la philosophie ricœurienne.

Nous arrivons cependant au point le plus difficile: le rapport entre l' "affirmation" ricœurienne et la "pulsion" freudienne - comprise cette fois comme pulsion de vie. Remarquons d'abord que la pulsion (Trieb) n'est pas le désir (Wunsch): la première est définie par son énergie, le second par sa visée; cette visée, en outre, dépend originellement de situations qui impliquent l'existence d'autrui; c'est pourquoi, quand la pulsion reste une poussée aveugle, le désir a la forme de la demande. Or Ricœur, lui, franchit cette ligne de partage: il fait descendre dans la pulsion la forme du désir. "La pulsion," déclare-t-il ainsi dans La critique et la conviction, "est adressée à"; elle exprime une relation à l'autre qui est "véritablement constitutive." ${ }^{33}$ Cette 
redéfinition de la pulsion la rend homogène à l'idée qu'il se fait de l'affirmation originaire, dans la mesure où celle-ci implique tout aussi originairement l'approbation d'autrui. Elle n'ajoute rien, cependant, à ce qu'on pouvait lire déjà dans l'Essai sur Freud: le fondateur de la psychanalyse "ne cherche pas la pulsion dans quelque vouloir-vivre inscrit en chacun: dans le vivant seul, il ne trouve que la mort"; "ce qui lutte contre la mort [...] est la conjugaison d'un mortel avec un mortel"; "c'est cela Eros [...]; c'est toujours avec un autre que le vivant lutte contre la mort." ${ }^{\prime 34}$

De là deux conséquences: a) la vie est humaine avant d'être médiatisée par le récit; b) elle s'affirme, dès ce moment, comme une vie partagée. Ce ne sont pas tant, cependant, les écrits théoriques de Ricœur, que ceux qu'on peut lire comme des témoignages, qui vérifient ces propositions. Dans son opus posthume, Vivant jusqu'à la mort, il évoque ainsi la "grâce intérieure" qui distingue l'agonisant du moribond; et il parle juste après de la mobilisation, dans sa personne, des "ressources les plus profondes de la vie à s'affirmer encore." 35 Demandera-t-on d'où viennent ces ressources? La réponse est peut-être dans ce mot: grâce. Car il peut signifier seulement la grâce de l'autre. C'est ce que suggère encore, dans le même opus, le commentaire ébauché dans la marge du texte où Jorge Semprun évoque l'agonie de Maurice Halbwachs à Buchenwald. Semprun: "j'avais pris la main de Halbwachs qui n'avait pas eu la force d'ouvrir les yeux; j'avais senti simplement une réponse de ses doigts, une pression légère: message presque imperceptible"; et Ricœur, à sa suite: "le donner-recevoir encore là." ${ }^{36}$

Que ce qui reste à la fin soit ce qui était au commencement, c'est ce qui autorise à parler non, certes, de l'énergétique mais de la dynamique ricœurienne - une dynamique réglée par le "donner-recevoir." C'est aussi ce qui explique la rencontre tardive de notre philosophe avec une psychanalyse différente de la psychanalyse freudienne, même si elle en dérive: celle de Heinz Kohut, le fondateur de la self-psychologie, à qui est consacré l'article le plus stimulant des "écrits et conférences" évoqués plus haut. ${ }^{37}$ Kohut adresse en effet à Freud trois reproches qui rejoignent les siens. Le premier concerne la notion d' "appareil psychique" avec ses "processus" et ses "mécanismes" en troisième personne, à quoi il oppose le concept global de "soi" (self). Le deuxième touche la fonction cognitive de la cure, chargée d'augmenter le savoir de soi, réduit luimême à un savoir d'origine verbale. Le troisième concerne cet idéal de libération par le savoir; car le besoin de savoir importe moins, en réalité, que celui d'empathie, une empathie qui englobe "toutes les modalités de l'aide qu'un soi peut apporter à un autre." "J'ai été très touché," commente Ricoeur, "par l'affirmation répétée de Kohut que l'homme a besoin jusqu'à son dernier souffle [...] du soutien d'un [autre soi] capable de l'aider à réaliser son projet de créativité intégrée." ${ }^{\prime 38} C^{\prime}$ est l'expérience du transfert qui forme alors, plus clairement encore que chez Freud, le cœur de la psychanalyse - une expérience qui enseigne que l'autonomie n'est possible que "par le moyen de l'hétéronomie." ${ }^{\prime 39}$ Comment mieux dire que le maintien de soi requiert la grâce de l'autre? Il est remarquable que Ricœur reçoive ces thèses comme une "leçon" que seule la psychanalyse peut procurer à la philosophie. Seule la psychanalyse? Ce n'est pas ce que montre la fin de l'article, qui fait entrer ces thèses en résonance avec différentes approches phénoménologiques de l'intersubjectivité.

Nous ne saurions présenter ici ces approches. Nous voudrions prévenir seulement l'étonnement que pourrait susciter ce pont jeté entre la psychanalyse et la phénoménologie. Ricœur n'a-t-il pas répété, à l'époque où il écrivait l'Essai sur Freud, que la psychanalyse était l'un des motifs de sa conversion à l'herméneutique? Mais la phénoménologie que l'on retrouve n'est pas celle que l'on avait accepté de perdre. Et c'est un nouveau chantier qu'ouvre Ricœur trente après la publication de l'Essai lorsqu'il déclare qu'il "n'avait pas pris assez au sérieux, à cette époque, les aspects de la phénoménologie en rapport avec la passivité: les synthèses passives, 
notamment," ${ }^{\prime \prime 0}$ et appelle à cette collaboration entre la phénoménologie et la psychanalyse. Il appartient à celles-ci d'explorer ensemble les formes primitives du donner et du recevoir, et de montrer comment elles préparent l'entrée en scène du langage et du récit.

\section{Conclusion}

En parlant, au seuil de cette étude, de l'inflation du récit, nous avions à l'esprit le titre d'un livre déjà ancien de Gilbert Hottois - bien qu'il ne traite pas seulement du récit: L'inflation du langage dans la philosophie contemporaine. ${ }^{41}$ Cette inflation est la preuve, selon Hottois, de la cécité du philosophe - homme du langage - devant la croissance absurde de la technoscience, qu'elle dénie en l'inversant. L'herméneutique de Gadamer en est à ses yeux le meilleur exemple. Il lui reproche ce qui constitue, pour une telle philosophie, le préjugé des préjugés: l'identité de l'être et du sens. Or il ne semble pas qu'une telle critique puisse être appliquée à l'herméneutique du Ricœur ${ }^{42}$, en dépit de son extrême attention aux puissances du langage et particulièrement du récit - même lorsque celle-ci paraît impliquer l'identité du vivre et du raconter. L'écart, entre les deux herméneutiques, ne tient pas à la place que trouverait dans la seconde la réflexion sur la technoscience: cette réflexion manque et l'on a dit plus haut, à propos de la crise de la narration, l'opposition de Ricœur à toute conception radicale en cette matière. Il grandit bien plutôt à mesure que s'infléchit une interrogation sur le mal où la souffrance vient progressivement prendre la place occupée auparavant par la faute, entendue comme une possibilité relative, dans son principe, à notre pouvoir pratique. Non que la souffrance ne résulte souvent de la faute et plus généralement de la violence, si l'on désigne par ce mot le mal que l'homme fait à l'homme: le combat éthique et politique trouve ici sa raison d'être. Mais il existe une souffrance qui ne vient pas de la violence. ${ }^{43}$ L'affinité de la narration et de l'action autorise alors à conclure des limites du récit à celles de l'action elle-même.

Mais c'est aux limites de l'herméneutique, encore une fois, que nous sommes conduits du même coup. Ricœur l'admet. La souffrance est pour lui un trou noir autour duquel gravitent les significations et qui toujours menace de les entraîner dans sa nuit. On peut certes s'étonner, s'il en est ainsi, de ses multiples citations du vers 177 de l'Agamemnon d'Eschyle: pathei mathos - souffrir pour comprendre. Mais ce qui est compris alors est précisément que tout ne peut être compris. Ce sont, au mieux, les conditions d'une compréhension que la souffrance espère mais dont elle éprouve actuellement l'impossibilité. Ce "comprendre" paradoxal est requis par tous les usages du récit. Il défend chacun de ce qui ne serait, sans cela, que l'illusion du sens. 
${ }^{1}$ Cf. Christian Salmon, Storytelling (Paris: La Découverte, 2007).

2 Jean-Marc Ferry, Les puissances de l'expérience 1: Le sujet et le verbe (Paris: Cerf, 1991).

3 Paul Ricoeur, "L'identité narrative," Esprit, 7-8 (1988), 310. Une critique à laquelle concourent selon lui l'interprétation et l'argumentation et qui obéit donc à des règles plus complexes que celles que lui suppose son interlocuteur. Selon ces règles, et comme l'implique précisément l'idée d'une "herméneutique critique," c'est l'argumentation qui est comprise finalement comme un moment de l'interprétation et non l'inverse.

4 Paul Ricœur, Temps et récit 3 (Paris: Seuil, 1985), 358.

5 Ricœur, Temps et récit 3, 359.

${ }^{6}$ La Symbolique du mal en offre déjà l'exemple par son exploration du langage de I'aveu, compris comme aveu de la faute.

7 Paul Ricœur, Du texte à l'action (Paris: Seuil, 1986), 40.

8 Thierry Hentsch, Raconter et mourir (Paris: Bréal, 2002).

9 La tragédie, l'épopée, le conte, la fable, le roman, la biographie, I'historiographie, entre autres, témoignent de cet art de raconter.

${ }^{10}$ Cf. en particulier Ricœur, Temps et récit 3, IIème partie, ch. 5: "L'entrecroisement de l'histoire et de la fiction."

11 Paul Ricœur, Temps et récit 1 (Paris: Seuil, 1983), 18.

12 Ricœur, Temps et récit $1,71$.

13 L'identité assignée par le récit l'est également, il est vrai, aux individus et aux communautés historiques. D'où les deux exemples que Ricœur met en parallèle dans les dernières pages de Temps et récit, où cette notion est introduite pour la première fois: celui de l'analysant - au sens qu'a ce terme en psychanalyse - et celui de I'Israël biblique. Dans les deux cas, écrit-il, "un sujet se reconnaît dans I'histoire qu'il se raconte à lui-même sur lui-même." Nous nous en tiendrons cependant à l'identité personnelle, dont la conception narrative est l'objet propre des Vème et VIème études de Soi-même comme un autre.

14 Ricœur, Temps et récit 3, "Conclusions," passim.

15 La logique du récit n'est pas celle du concept. Une histoire plus intelligible n'est pas une histoire intégralement intelligible. Dans un chapitre intitulé sobrement: "Renoncer à Hegel," Ricœur se demande si l'histoire philosophique - cette "histoire pensante" dont Hegel prétend recueillir le sens, est écrite par Dieu ou par les hommes; il interprète le schème de la ruse de la raison comme l'expression d'une "théologie honteuse" et montre que cette théologie réduit le temps à un éternel présent. La totalisation opérée par I'histoire philosophique abolit ainsi la possibilité même d'une histoire en général. Certes, on ne réfute pas Hegel. Mais "renoncer" n'est pas réfuter. C'est dire simplement: nous ne pensons plus comme cela. C'est un fait: la philosophie hégélienne de I'histoire nous apparaît elle-même comme un moment dépassé de la conscience que nous avons de nous- 
mêmes. Nous l'avons laissée derrière nous plus sûrement que Hegel n'avait laissé derrière lui tous ceux qui avaient compté à ses yeux dans I'Histoire universelle. Il se peut qu'ainsi nous sachions moins. Mais nous comprenons plus. C'est pourquoi Ricœur oppose finalement, à la philosophie hégélienne de I'histoire, une "herméneutique de la condition historique."

16 Paul Ricœur, "Mythes du salut et raison," Ecrits et conférences 2: Herméneutique (Paris: Seuil, 2010), 271 et suivantes.

17 On a peut-être là un début de réponse à l'objection formulée par la Critique des idéologies contre la conception narrative de l'identité, suspecte de sacrifier la raison à la tradition et l'humanité à la communauté.

${ }^{18}$ Auxquels nous sommes tentés d'appliquer librement la distinction que fait Bergson entre sociétés closes et sociétés ouvertes.

19 Walter Benjamin, "Le Narrateur," réédité dans Rastelli raconte et autres récits, trad. M. de Gandillac (Paris: Seuil, 1987), 143 et suivantes.

${ }^{20}$ Ricœur, Temps et récit 2 (Paris: Seuil, 1984), 48.

${ }^{21}$ Sarah Kofman, Paroles suffoquées (Paris: Galilée, 1987), 16.

22 Paul Ricœur, Soi-même comme un autre (Paris: Seuil, 1990), 370.

23 Ricœur, Temps et récit 3, 390.

24 Objectera-t-on que cette suspension même annonce une nouvelle institution du sens, à la façon dont opère la métaphore, dont le modèle serait ainsi transposable au récit? Mais ce qui est vrai sur le plan de la préfiguration, l'est aussi sur celui de la configuration et de la refiguration: I'imagination narrative manque pour la souffrance des ressources qu'elle déploie pour la faute.

25 Ricœur, Soi-même comme un autre, 370.

26 Ricœur, Soi-même comme un autre, 370. Voir en outre Ricœur, "Multiple étrangeté," Fremdheit und Vertrauheit. Hermeneutik im Europaïschen Kontext, (Leuven: Peeters, 2000).

27 Pour une présentation développée des remarques qui suivent, nous nous permettons de renvoyer à notre article, J. Porée, "La philosophie au miroir de la psychanalyse," Laval théologique et philosophique 65/3 (2009), 405-430.

28 Cette conception narrative de la psychanalyse, presque toujours bienveillante, diffère de celles que I'on trouve dans Le volontaire et l'involontaire et dans I'Essai sur Freud.

29 Ricœur, Ecrits et conférences 1: Autour de la psychanalyse (Paris: Seuil, 2008).

30 Ricœur, Ecrits et conférences 1: Autour de la psychanalyse, 257 et suivantes.

31 Particulièrement I'interprétation lacanienne.

32 Ricœur, "Psychanalyse et herméneutique," dans Ecrits et conférences 1: Autour de la psychanalyse, 87. 
33 Paul Ricœur, La critique et la conviction (Paris: Calmann-Lévy, 1995), 111.

34 Paul Ricœur, De l'interprétation. Essai sur Freud (Paris: Seuil, 1965), 287; c'est Ricœur qui souligne.

35 Paul Ricœur, Vivant jusqu'à la mort (Paris: Seuil, 2007), 43.

36 Ricœur, Vivant jusqu'à la mort, 49.

37 "Le self selon la psychanalyse et selon la philosophie phénoménologique," Ecrits et conférences 1: Autour de la psychanalyse, 139 et suivantes. Voir sur ce point, outre notre article cité supra, note 27, I'article de Michel Dupuis, "L'empathie comme outil herméneutique du soi. Note sur Paul Ricoeur et Heinz Kohut", ERSS, 1/1, (2010), 9-20.

38 Paul Ricœur, "Le self selon la psychanalyse et selon la philosophie phénoménologique," Ecrits et conférences 1: Autour de la psychanalyse, 140-141.

39 Ricœur, "Le self selon la psychanalyse et selon la philosophie phénoménologique," 152.

40 Ricœur, La critique et la conviction, 112-113.

41 Gilbert Hottois, L'inflation du langage dans la philosophie contemporaine, Editions de I'Université de Bruxelles, 1979.

42 Hottois, lui, ne semble pas faire de différence.

43 Cf. sur ce point, comme sur le précédent, Paul Ricœur, Le mal. Un défi à la philosophie et à la théologie (Genève: Labor et Fides, 1986), 44. 